\title{
Éditorial
}

\section{Changements climatiques et santé : un grand défi et une grande chance pour la santé publique au Canada}

\author{
Ashlee Cunsolo, Ph. D. (1); Sherilee L. Harper, Ph. D. (2)
}

Les changements climatiques ont un impact profond sur la santé humaine partout dans le monde ${ }^{1,2}$. Ils affectent directement la santé humaine en provoquant des phénomènes météorologiques extrêmes (vagues de chaleur, sécheresses, pluies torrentielles, etc.) qui entraînent une hausse de la morbidité et de la mortalité liées à la chaleur et au froid, des blessures non intentionnelles et des décès, ainsi que d'autres conséquences indésirables. Les changements climatiques ont également une influence indirecte sur la santé, par leurs effets sur les écosystèmes et les systèmes humains, qui se répercutent sous forme de maladies d'origine alimentaire et hydrique, de maladies à transmission vectorielle, de maladies respiratoires, de défis sur le plan de la santé et de la sécurité au travail, de dénutrition, ainsi que de problèmes de santé mentale et de bien-être ${ }^{1,2}$.

Les conséquences des changements climatiques sur la santé humaine ne sont ni réparties ni vécues uniformément. Une myriade de facteurs politiques, culturels, économiques, institutionnels, géographiques et démographiques, généralement interreliés, déterminent l'impact qu'auront ou non les changements climatiques sur la santé, notamment l'exclusion sociale, les inégalités et les écarts en matière de disponibilité et de contrôle des ressources sociales, financières et environnementales nécessaires à l'adaptation et à la résilience ${ }^{2}$. En raison des multiples facettes, de l'étendue et de la complexité de ces impacts sur la santé humaine, l'un des défis marquants de notre époque est de déterminer les conséquences des changements climatiques sur la santé humaine ainsi que les mesures à prendre face à celles-ci. Ce défi a déjà été souligné par deux commissions du Lancet $^{1,3}$, par l'Organisation mondiale de la santé $(\mathrm{OMS})^{4}$ et par l'Association médicale canadienne $^{5}$, ainsi qu'aux États-Unis ${ }^{6}$ et au Royaume-Uni? .

Même si les dangers des changements climatiques pour la santé sont clairs et urgents $^{2,3}$, les changements climatiques sont de plus en plus présentés comme une occasion à saisir pour la santé publique ${ }^{1}$. Par exemple, les effets néfastes des changements climatiques sur la santé pourraient être réduits par l'augmentation de l'offre en soins de santé et en services de santé publique, l'amélioration de la gestion des catastrophes, l'intégration de la surveillance de l'environnement et de la santé et des systèmes d'alerte précoce, la réduction de la pauvreté et le développement des collaborations intersectorielles ${ }^{2}$. L'atténuation des changements climatiques comporte aussi d'autres avantages connexes pour la santé, par exemple l'amélioration de l'activité physique par l'adoption de modes de transport actifs ou encore la réduction des maladies respiratoires par la diminution de la pollution atmosphérique ${ }^{2}$. Bien que le secteur de la santé doive déjà composer avec les conséquences des changements climatiques sur la santé et sur les besoins, les priorités, les consultations, les interventions et les coûts connexes, l'adaptation du secteur de la santé est généralement sous-représentée dans les politiques, la planification et les programmes. Par exemple, toutes les initiatives prises en vertu de la Convention-cadre des Nations Unies sur les changements climatiques (CCNUCC) touchent la santé humaine, mais seulement $15 \%$ d'entre elles comportent un volet explicite en la matière ${ }^{8}$
Le Canada ne fait pas exception à cette tendance mondiale : le secteur de la santé y est nettement sous-représenté dans les initiatives d'adaptation en comparaison des autres secteurs ${ }^{8}$. La majorité des interventions gouvernementales visant à adapter le secteur de la santé sont des travaux préparatoires concentrés sur l'établissement des moyens d'adaptation et la mise en place des conditions favorables à cette adaptation, en particulier la sensibilisation aux conséquences des changements climatiques sur la santé et la réalisation d’évaluations de la vulnérabilitée-10.

Les gouvernements prennent de plus en plus de mesures d'adaptation pour réduire la vulnérabilité, par exemple la mise en place de systèmes de surveillance et d'alerte, ainsi que des initiatives visant à modifier les pratiques et les comportements. Au Canada, ces actions prennent la forme de formations, de ressources d'information, de cadres, de mesures générales de sensibilisation et d'éducation et de transmission de l'information aux décideurs'. Il importe de noter que l'adaptation du secteur de la santé est en train de se faire à l'échelle locale, que ce soit à l'échelle de l'individu, du ménage ou de la collectivité locale.

À la lumière des lacunes en matière de recherche, des possibilités et des difficultés concernant la pratique de la santé publique et de l'urgence des conséquences des changements climatiques sur la santé publique, les six contributions à ce numéro spécial, publié de concert avec le Jour de la Terre, illustrent les nombreuses manières dont les changements climatiques ont déjà une influence sur la santé à l'échelle individuelle et collective. Cet ensemble illustre, 
par une combinaison d'aperçus et d'articles de recherche originaux, la diversité de la recherche sur les changements climatiques et la santé au Canada. Il constitue un appel à l'action clair à l'intention du secteur de la santé, qui doit à la fois soutenir les mesures d'adaptation aux changements climatiques et promouvoir les stratégies d'atténuation qui présentent des avantages connexes clairs pour la santé.

Ce numéro spécial commence par une synthèse des problèmes de santé liés au climat qui ont été recensés par Kipp et ses collaborateurs dans les régions rurales et éloignées du Canada ${ }^{11}$. Cet article, qui est fondé sur un examen de la portée, fait ressortir les principaux points forts et points faibles des régions rurales et éloignées sur le plan de la santé. Il porte sur les problèmes affectant la sécurité alimentaire et l'eau, l'exacerbation des maladies chroniques, les maladies infectieuses, la morbidité et la mortalité ainsi que la santé mentale.

Les populations autochtones (membres des Premières Nations, Inuits et Métis) subissent de façon disproportionnée les conséquences des changements climatiques sur la santé et le bien-être. Richards et ses collaborateurs $^{12}$ décrivent le Programme sur le changement climatique et l'adaptation du secteur de la santé (PCCASS) de la Direction générale de la santé des Premières Nations et des Inuits de Services aux Autochtones Canada, qui finance la recherche sur l'adaptation des collectivités dans le but d'arriver à des outils et à des solutions locales qui soient adaptés à la réalité culturelle. Les auteurs, qui ont utilisé trois études de cas pour présenter la diversité des études financées par ce programme, soulignent l'importance non seulement de financer la recherche sur les changements climatiques et la santé mais aussi d'aider directement les collectivités autochtones à réaliser leurs propres recherches, en harmonie avec leurs contextes locaux et leurs cultures.

Soulignant la grande importance des avantages connexes, Kingsley ${ }^{13}$ s'intéresse aux manières dont la protection, l'amélioration et l'agrandissement des espaces verts peuvent non seulement constituer une stratégie d'atténuation des changements climatiques, mais aussi une stratégie clé en matière de santé qui présenterait des bienfaits contre les maladies chroniques et leurs facteurs de risque. Fondée sur deux exemples de collaborations multisectorielles, auxquelles ont participé notamment EcoHealth Ontario et Climate Change Parks en Écosse, cette contribution propose un modèle dans lequel les espaces verts sont considérés comme une intervention importante tant pour la lutte contre les changements climatiques que pour la santé.

Demers et Gosselin ${ }^{14}$ traitent de la hausse des allergies aux pollens et des rhinites allergiques saisonnières en Amérique du Nord à la suite des changements climatiques, particulièrement en lien avec l'herbe à poux. Dans leur article, les auteurs analysent une intervention du gouvernement du Québec visant à réduire les pollens d'herbe à poux et d'autres plantes allergènes. L'examen des données scientifiques sur laquelle s'appuyait l'intervention indique que celle-ci s'est révélée efficace dès sa mise en œuvre.

MacIntyre et ses collaborateurs ${ }^{15}$ examinent les modes de communication des risques liés aux phénomènes météorologiques extrêmes et aux changements climatiques dans le secteur de la santé publique. Les auteurs ont réalisé un examen de la portée pour évaluer les stratégies de communication des risques en santé publique puis pour mesurer les implications de leurs résultats au regard des Normes de santé publique de l'Ontario de 2018. Ces résultats, qui indiquent que la communication des risques est plus efficace si elle porte sur des phénomènes météorologiques extrêmes à court terme, vont contribuer à orienter les stratégies de communication en santé publique et à favoriser la prise de mesures locales pour atténuer les effets des changements climatiques.

Enfin, Malena-Chan ${ }^{16}$ a utilisé une approche narrative pour étudier les manières dont les jeunes leaders communautaires en Saskatchewan interprètent et comprennent les changements climatiques. Les résultats de cette étude laissent penser que la dissonance narrative - c'est-à-dire l'incapacité de concilier plusieurs visions des changements climatiques en raison de contradictions émotionnelles, morales ou conceptuelles - est un facteur qui pourrait expliquer l'immobilisme et l'inaction, et qui nécessiterait donc une adaptation des messages sur la santé publique.

En 2015, la Commission du Lancet sur les changements climatiques et la santé a présenté aux différents États dix recommandations les pressant d'" investir dans la recherche sur les changements climatiques et la santé publique ", de " financer des systèmes de santé pouvant faire face aux changements climatiques » et de « collaborer pour mettre en place des politiques qui atténueront les changements climatiques et favoriseront la santé publique, et surveiller l'évolution de la situation durant les 15 prochaines années ${ }^{17}$. Ce présent numéro spécial, qui s'appuie sur ces recommandations et sur un corpus de plus en plus important de publications scientifiques sur le sujet, montre la diversité des effets des changements climatiques sur la santé de la population canadienne tout en faisant ressortir les grands défis et les grandes occasions que présentent les changements climatiques.

\section{Conflits d'intérêts}

Les auteures déclarent n'avoir aucun conflit d'intérêts.

\section{Contributions des auteures et avis}

AC et SLH ont contribué de façon égale à la conception, la rédaction et la révision de cet article.

Le contenu de l'article et les points de vue qui y sont exprimés n'engagent que les auteures; ils ne correspondent pas nécessairement à ceux du gouvernement du Canada.

\section{Références}

1. Wang H, Horton R. Tackling climate change: the greatest opportunity for global health. Lancet. 2015;386(10006): 7-13.

2. Smith KR, Woodward A, CampbellLendrum D, et al. Human health: impacts, adaptation, and co-benefits. Dans Field CB, Barros VR, Dokken DJ, et al. (dir.), Climate Change 2014: Impacts, Adaptation, and Vulnerability Part A: Global and Sectoral Aspects Contribution of Working Group II to the Fifth Assessment Report of the Intergovernmental Panel on Climate Change. London (UK) : Cambridge University Press; 2014. p. 709-754.

3. Costello A, Abbas M, Allen A, et al. Managing the health effects of climate change. Lancet. 2009;373(9676):16931733 . 
4. Organisation mondiale de la santé. COP24 special report: health and climate change. Genève (Suisse) : Organisation mondiale de la santé; 2018. 38 p.

5. Association médicale canadienne. Les changements climatiques et la santé humaine. Énoncé de politique de l'Association médicale canadienne [Internet]. Ottawa (Ont.) : Association médicale canadienne; 2010. En ligne à : http://policybase.cma.ca/dbtw-wpd /Policypdf/PD10-07F.pdf

6. American Medical Association. Confronting health issues of climate change [Internet]. American Medical News. 2011. En ligne à : https:// amednews.com/article/20110404 /opinion/304049959/4/\#exli

7. British Medical Association. BMA backs action on health effects of climate change [Internet]. British Medical Association; 2015. En ligne à : https:// www.bma.org.uk/news/2015/october /bma-backs-action-on-health-effects -of-climate-change

8. Lesnikowski AC, Ford JD, BerrangFord L, Paterson JA, Barrera M, Heymann SJ. Adapting to health impacts of climate change: a study of UNFCCC Annex I parties. Environ Res Lett. 2011;6(4):044009.

9. Austin S, Ford J, Berrang-Ford L, Araos M, Parker S, Fleury M. Public health adaptation to climate change in Canadian jurisdictions. Int $\mathrm{J}$ Environ Res Public Health. 2015;12(1):623-651.

10. Panic M, Ford JD. A review of nationallevel adaptation planning with regards to the risks posed by climate change on infectious diseases in 14 OECD nations. Int $\mathrm{J}$ Environ Res Public Health. 2013;10(12):7083-7109.

11. Kipp A, Cunsolo A, Vodden K, et al. Aperçu - Les effets des changements climatiques sur la santé et le bien-être dans les régions rurales et éloignées à travers le Canada : synthèse documentaire. Promotion de la santé et prévention des maladies chroniques au Canada. 2019;39(4):134-139.
12. Richards G, Frehs J, Myers E, Van Bibber M. Commentaire - Le Programme sur le changement climatique et l'adaptation du secteur de la santé : mesures d'adaptation mises en avant par les chefs de file autochtones en matière de climat. Promotion de la santé et prévention des maladies chroniques au Canada. 2019;39(4):140-143.

13. Kingsley M, EcoHealth Ontario. Commentaire - Changements climatiques, santé et avantages connexes des espaces verts. Promotion de la santé et prévention des maladies chroniques au Canada. 2019;39(4):144-148.

14. Demers I, Gosselin P. Aperçu - Pollens, climat et allergies : initiatives menées au Québec. Promotion de la santé et prévention des maladies chroniques au Canada. 2019;39(4):149-154.

15. MacIntyre E, Khanna S, Darychuk A, Copes R, Schwartz B. Synthèse des données probantes - Évaluation de la communication des risques en présence de phénomènes météorologiques extrêmes et des changements climatiques : examen de la portée. Promotion de la santé et prévention des maladies chroniques au Canada. 2019; 39(4):155-171.

16. Malena-Chan R. Modélisation narrative pour étudier l'engagement à l'égard de la lutte contre les changements climatiques chez de jeunes dirigeants communautaires. Promotion de la santé et prévention des maladies chroniques au Canada. 2019;39(4): 172-182.

17. Watts N, Amann M, Ayeb-Karlsson S, et al. The Lancet Countdown on health and climate change: from 25 years of inaction to a global transformation for public health. The Lancet. 2018;391(10120):581-630. 